direct cholinergic stimulation of the hypothalamus. Nature, $1969,224,1035-1036$.

Bandler, R. J., Jr. Cholinergic synapses in the lateral hypothalamus for the control of predatory aggression in the rat. Brain Research, 1970, 20, 409-424.

Bandler, R. J., Jr. Direct chemical stimulation of the thalamus: Effects on aggressive behavior in the rat. Brain Research, 1971 a, 26, 81-93.

Bandler, R. J., Jr. Chemical stimulation of the rat midbrain and aggressive behavior. New Biology, 1971b, 229, 222-223.

Bandler, R. J., Jr., \& Moyer, K. E. Animals spontaneousiy killed by rats. Communications in Behavior Biology, 1970, 5 , 177-182.

Karli, P. The Norway rat's killing response to the white mouse. Behavior, 1956, 10, 81-103.

Karli, P., Vergnes, M.. \& Didiergeorges, F. Rat-mouse interspecific aggressive behavior and its manipulation by brain ablation and brain stimulation. In E. B. Sigg and S. Garattini (Eds.), Biology of aggressive behavior. Amsterdam: Excerpta
Medica Foundation, 1969. Pp. 47-55.

King, M. B., \& Hoebel, B. G. Killing elicited by brain stimulation in rats. Communications in Behavioral Biology, 1968, 2, 173-177.

Moyer, K. E. Kinds of aggression and their physiological basis. Communications in Behavioral Biology, 1968, 2, 66-87.

Panksepp, J. Aggression elicited by electrical stimulation of the hypothalamus in albino rats. Physiology \& Behavior, 1971, 6, 321-329.

Smith, D. E King, M, B. \& Hoebel, B. G. Lateral hypothalamic control of killing: Evidence for a cholinoceptive mechanism. Science, 1970, 167, 900-901.

Vergnes, M., \& Karli, P. Effets de la stimulation de l'hy pothalamus lateral, de l'amygdale et de l'hippocampe sur le comportement d'agression interspecifique Rat-Souris. Physiology \& Behavior, 1969, 4, 889-894.

(Received for publication March 12, 1973.)

\title{
Information processing in the visual periphery*
}

\author{
JAMES R. ANTES and DAVID C. EDWARDS \\ Iowa State University, Ames, Iowa 50010
}

The information processing capacity of the visual periphery was investigated in two experiments using stimuli of known relative information content. The results of Experiment I showed redundant patterns to be easiest to identify at all peripheral angles tested $(5,10$, 20 , and $30 \mathrm{deg}$ ) as compared to intermediate and nonredundant patterns. Performance on all patterns decreased as peripheral angle increased. In Experiment II, simultaneous foveal and peripheral vision was required in a discrimination task. Performance was above chance only when the peripheral (7-deg) pattern was redundant. The foveal pattern had no effect on discrimination.

Many researchers attempting to describe visual information processing have dichotomized the regions of visual input into fovea and periphery. Typically they have characterized the foveal region as the source of almost all information input, ascribing only crude processing ability to the periphery (e.g., Gibson, 1966; Neisser, 1967; Williams, 1966). The bulk of the research, however, supports the notion of a structural and

*Portions of this research were reported at the 1970 Psychonomic Society meeting. functional continuum from the fovea to the extreme periphery. Polyak (1941) distinguished seven retinal regions based on changes within layers of the retina, emphasizing that these areas are not abruptly separated but blend into each other. Since the research of Wertheim (1894), many investigators have reported a continuous decrement in visual acuity with increasing peripheral angle. Numerous others have reported a similar decline in other measures of visual performance with peripheral stimulus presentation.

One important functional question concerns the relative efficacy of a dichotomous vs a continuous model of visual field in guiding future research on visual information processing. It appears certain that a more comprehensive model of visual search, for example, is possible if a continuum of visual field input is assumed. The research reported here is a preliminary investigation of peripheral information processing. Much is known about the acuity of the periphery and its capacity to detect geometric forms (e.g., Geissler, 1926; Menzer \& Thurmond, 1970). However, little is known about peripheral processing of stimuli varying on a redundancy dimension. Quantification of processing capabilities across the retina appears to be an important first step in modifying the model of visual field input. The following two experiments are initial attempts to investigate 


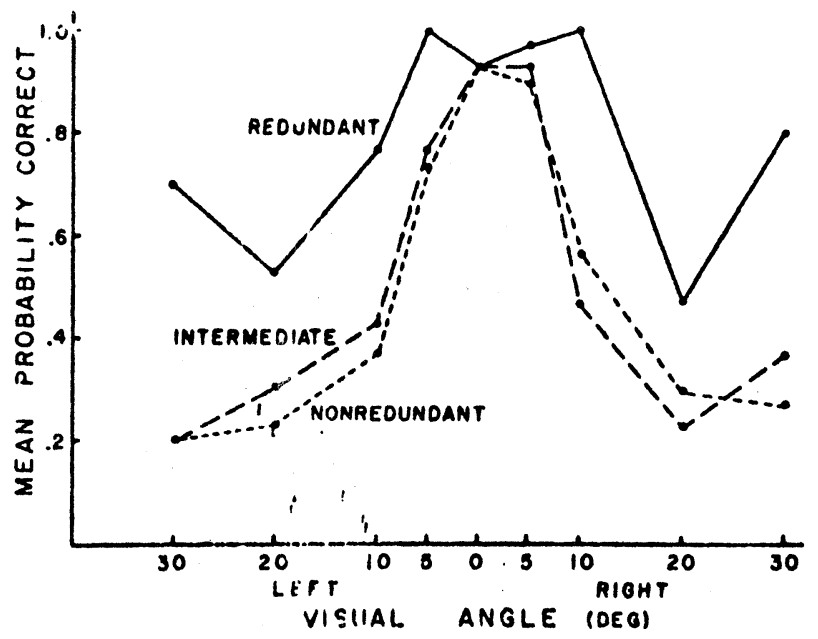

Fig. 1. Mean probability of completely correct identification at all peripheral locations for each information level.

peripheral processing of stimuli that vary on an information scale of ordinal properties.

\section{EXPERIMENT I}

Stimuli

Garner has developed a series of five-dot patterns generated from a nine-dot space ( 3 by 3 matrix). He has shown that redundancy of the patterns, as determined by the size of the subset of reflection and rotation to which a pattern belongs, shows a strong relationship to rated "goodness" (Garner \& Clement, 1963). The three reflection and rotation subsets, redundant $(\mathrm{R})$, intermediate (I), and nonredundant $(\mathrm{N})$, were rated good, intermediate, and poor, respectively. Two $\mathrm{R}$, two I, and two $\mathrm{N}$ patterns were chosen as stimuli and were photographed, white on black, and mounted in slide carriers. Each stimulus pattern subtended a visual angle of $2.5 \mathrm{deg}$ horizontally and vertically.

\section{Subjects}

The Ss were two male graduate students from the Iowa State University Psychology Department and one local male professional pilot.

\section{Procedure}

The $\mathrm{S}$ was seated with his head positioned on a chinrest facing a rear projection screen and was instructed to fixate a central cross. After a "ready" signal from E, each stimulus was projected onto the screen for $200 \mathrm{msec}$. $S$ was instructed to maintain fixation on the cross and, when the stimulus appeared, to use peripheral vision to identify the pattern. The $S$ was supplied with an individual slip of paper for each trial, upon which was printed a facsimile 3 by 3 matrix. After each trial, $S$ marked the five dots he saw and handed the slip to E. Visual angles tested were 0 (foveal) and 5, 10, 20, and 30 deg left and right. Each slide was presented five times at each angle in a random order. The first 30 trials were presented at $30 \mathrm{deg}$ left, then $30 \mathrm{deg}$ right, $20 \mathrm{deg}$ left, etc., with a short rest between each block of 30 trials. The $S$ was informed of the peripheral location for each block of trials. The right eye was covered for trials on the left, the left eye was covered for trials on the right, and both eyes remained open for foveal trials.

\section{Results}

Figure 1 shows the mean probability of completely correct identification at each peripheral location for each information level. The number of correct scores was subjected to a 4 by 3 by 3 by 2 factorial analysis of variance, with peripheral angle (excluding foveal), Ss, redundancy, and side (left vs right) as main effects. Three main effects and no interactions were significant: peripheral angle, $\mathrm{F}(3,46)=21.58, \mathrm{p}<.01$; redundancy, $\mathrm{F}(2,46)=17.75, \mathrm{p}<.01 ;$ and $\mathrm{Ss}, \mathrm{F}(2,46)=30.04$, $\mathrm{p}<.01$. Post hoc comparisons using Newman-Keuls procedures (alpha $=.05$ for all comparisons) showed performance at $5 \mathrm{deg}$ to be superior to that at the other locations, at $10 \mathrm{deg}$ to be better than that at 20 and $30 \mathrm{deg}$, and no difference in performance at the latter two locations. Ss showed superior identification for the $\mathrm{R}$ patterns, with no difference between the $\mathrm{N}$ and $\mathrm{I}$ patterns.

Briefly, the results demonstrate that Ss performed better at all peripheral locations up to $30 \mathrm{deg}$ on redundant figures as compared to nonredundant and intermediate patterns. In addition, performance for all information loads decreased with increasing peripheral angle up to $20 \mathrm{deg}$. Thus, using stimuli of known relative information content, it was shown that the periphery has a limited information gathering capacity and that this capacity becomes smaller with increased peripheral distance.

The above experiment confounded peripheral angle with blocks of trials. Consequently, the study was replicated using 10 additional Ss, who were given no prior information regarding the peripheral location of the stimuli (three of the $\mathrm{R}$ and three of the $\mathrm{N}$ patterns). ${ }^{1}$ The order of presentation was random with respect to peripheral location and redundancy. Again peripheral angle and redundancy were significant sources of variation. The probability correct scores matched those of the previous experiment closely, except for a depression in foveal detection of nonredundant patterns. Thus, the significant peripheral angle effect in the previous experiment was not due to a practice effect.

Two further points deserve mention. First, the significant redundancy effect may not be attributed to differential acuity of the $\mathrm{R}$ vs $\mathbf{N}$ figures. All patterns were generated from the same nine-dot matrix, making physical size constant across the entire stimulus set. Second, because of the response technique employed here, a form of response bias may be responsible for some of the redundancy effect. Handel \& Garner (1966) have shown that patterns of greater redundancy tend to be produced as associates of less redundant patterns. That is, there is a tendency toward figural "goodness" when associating these patterns. In the present experiment, this bias would tend to spuriously inflate performance on the $\mathrm{R}$ patterns and diminish that on the $\mathrm{I}$ and $\mathrm{N}$ patterns. In the following experiment, a same-different matching task was employed to eliminate this bias.

\section{EXPERIMENT II}

The second experiment involved a situation in which 
simultaneous foveal vision and peripheral vision were necessary. Ss were required to indicate whether the two stimuli were the same or different.

\section{Stimuli}

The stimuli were three of the $\mathrm{N}$ and three of the $\mathrm{R}$ dot patterns devised by Garner (Garner \& Clement, 1963).

\section{Subjects}

The Ss were 17 undergraduates (9 males) enrolled in the general psychology course at Iowa State University. Research participation was a course requirement.

\section{Procedure}

The $\mathrm{S}$ was seated as before in front of the rear projection screen. On a "ready" signal from E, a pair of stimuli appeared for $200 \mathrm{msec}$, one foveally and the other at $7 \mathrm{deg}$ of visual angle in either the left or right hemifield at a 30-deg incline from horizontal. $\mathrm{S}$ was instructed to maintain fixation on the center of an outlined area on the screen, where the foveal stimuli were to appear. He was told to determine whether the two stimuli presented were the same or different and to make a discrimination response using a 6-point scale anchored as $1=$ different and $6=$ same. The rating scale was employed for signal detection analyses. The design was balanced such that each pattern was paired with every other pattern and all figures were presented an equal number of times at both the foveal and peripheral locations. The 48 trials were presented in a fixed random order to each S (24 additional trials in which both patterns were presented at peripheral locations were included in the original experiment but are not a part of this analysis).

\section{Results}

Partitioning the rating scale at $1-3=$ different and 4-6 = same, mean probability correct scores for the four experimental conditions were: fovea redundant/periphery redundant $\quad$ (FR-PR) $=.72$, FR-PN $=.45$, FN-PR $=.74$, FN-PN $=.61$. A balanced design was desirable for this analysis. Because same trials were possible for only two of the four conditions, a simple two-way classification analysis of variance was performed using number correct scores from the different trials only. An inspection of the results for the discarded same trials in the FR-PR and FN-PN conditions showed the probabilities correct to be similar to those for the different trials (FR-PR, .72 vs .71; FN-PN, .62 vs .58). The experimental conditions effect was highly significant, $F(3,48)=9.45, p<.01$. A Newman-Keuls post hoc comparison showed all conditions to be different from all others except for the FN-PR vs FR-PR comparison (alpha $=.05$ ). A chi-square test on the FR-PN and FN-PN scores demonstrated these values not to be significantly different from chance levels.

Briefly, then, the results showed superior performance when the peripheral stimulus was redundant, regardless of the foveal stimulus, and chance performance when the peripheral pattern was nonredundant. To test this result directly, all trials were partitioned into FR vs FN and PR vs PN groups. Probabilities correct and the signal detection sensitivity measure were, respectively: FR: $.65,0.815$; FN: .64, 0.718; PR: .72, 1.196; PN: .57, 0.331 . Paired $t$ tests on the number correct data and
$G$ tests (Gourevitch \& Galanter, 1967) on the d' data showed no differences in the FR vs FN comparisons: $\mathrm{t}(16)=1.645, \mathrm{p}>.10, \mathrm{G}=0.536, \mathrm{p}>.10$. However, highly significant differences obtained in the PR vs PN comparisons: $\mathrm{t}(16)=4.932, \mathrm{p}<.001, \mathrm{G}=4.75$, $\mathrm{p}<.001$. Thus, for this task the foveal pattern had little effect on performance; discrimination performance was determined by the relative information load in the periphery.

\section{DISCUSSION}

The data showed, first, that the visual periphery has a limited information gathering capacity and that this capacity diminishes with increasing peripheral distance. This common finding is, of course, related to factors determining acuity of detection. It was also shown that, when simultaneous use of the fovea and periphery ${ }^{2}$ is necessary, peripheral discrimination deteriorates and is only at a chance level with nonredundant peripheral stimuli. These redundancy effects did not depend upon size of stimulus. Acuity was held constant in these comparisons. Finally, Experiment II showed that the fovea has little effect on the discrimination of peripheral patterns. This last finding is apparently at odds with the results of Adams (1971), who demonstrated that foveal task difficulty affected peripheral task performance.

Taken together, these experiments have shown that there is a differential capacity for processing redundant and nonredundant information in the periphery. The second experiment provided support for the supposition that the redundancy effects of the first experiment were not due solely to a possible response bias. It is apparent that theoretical considerations must include a more sophisticated processing role for peripherally received stimulation.

\section{REFERENCES}

Adams, R. W. Peripheral vision and visual attention. Unpublished doctoral dissertation, Iowa State University, 1971.

Garner, W. R., \& Clement, D. E. Goodness of pattern and pattern uncertainty. Journal of Verbal Learning \& Verbal Behavior, 1963, 2, 446-452.

Geissler, L. R. Form perception in indirect vision. Psychological Bulletin, 1926, 23, 135-136.

Gibson, J. J. The senses considered as perceptual systems. Boston: Houghton Mifflin, 1966.

Gourevitch, V., \& Galanter, E. A significance test for one parameter isosensitivity functions. Psychometrika, 1967, 32 , 25-33.

Handel, S., \& Garner, W. R. The structure of visual patterns associates and pattern goodness. Perception \& Psychophysics, $1966,1,33-38$.

Menzer, G. W., \& Thurmond, J. B. Form identification in peripheral vision. Perception \& Psychophysics, 1970, 8, 205-209.

Neisser, U. Cognitive psychology, New York: Appleton-Century-Crofts, 1967.

Polyak, S. L. The retina. Chicago: University of Chicago Press, 1941 .

Wertheim, T. Uber die indireckte sehscharfe. Zeitschrift fur Psychologie und Physiologie der Sinnesorgane, 1894, 7,

172-187.
Williams, $L$. G. The effect of target specification on objects fixated during visual search. Perception \& Psychophysics, $1966,1,315-318$.

\section{NOTES}

1. The "T" pattern, although a member of the I subset of patterns, was rated third in goodness by Ss, ranking behind the two $R$ patterns (Garner \& Clement, 1963), and was considered to be a member of the $R$ subset for this and the following experiment.

2. The 7-deg locus actually lies in the perifovea, according to the neurologically defined taxonomy established by Polyak (1941).

(Received for publication March 12, 1973.) 